\title{
Pauci-immune glomerulonephritis
}

INSERM

\section{Source}

INSERM. (1999). Orphanet: an online rare disease and orphan drug data base. Pauciimmune glomerulonephritis. ORPHA:93126

Pauci-immune glomerulonephritis (GN) is one of the most frequent causes of rapidly progressive GN (RPGN, see this term). It is characterized clinically by renal manifestations of RPGN (hematuria, hypertension) leading to renal failure within days or weeks, and may be associated with manifestations of systemic vasculitis (arthralgia, fever, seizures, mono neuritis and lung involvement). Pauci-immune GN is histologically characterized by focal necrotizing and crescentic GN, with mild or absent glomerular staining for immunog lobulin and complement by fluorescence microscopy, which may manifest either as part of a systemic small vessel vasculitis (including microscopic polyang iitis, granulomatosis with polyang iitis and eosinophilic granulomatosis with polyang iitis (see these terms)), or rarely as part of renal-limited vasculitis (RLV, idiopathic crescentic GN). Immunologic classification is based on the presence or absence of circulating antineutrophil cytoplasmic antibodies (ANCAs), namely pauci-immune-GN with ANCA and pauci-immune GN without ANCA (see these terms). 